\title{
Mutant Cohesin in Premature Ovarian Failure
}

\author{
Sandrine Caburet, Ph.D., Valerie A. Arboleda, Ph.D., Elena Llano, Ph.D., \\ Paul A. Overbeek, Ph.D., Jose Luis Barbero, Ph.D., Kazuhiro Oka, Ph.D., \\ Wilbur Harrison, M.S., Daniel Vaiman, Ph.D., Ziva Ben-Neriah, M.D., \\ Ignacio García-Tuñón, Ph.D., Marc Fellous, M.D., Ph.D., Alberto M. Pendás, Ph.D., \\ Reiner A. Veitia, Ph.D., and Eric Vilain, M.D., Ph.D.
}

SUMMARY

Premature ovarian failure is a major cause of female infertility. The genetic causes of this disorder remain unknown in most patients. Using whole-exome sequence analysis of a large consanguineous family with inherited premature ovarian failure, we identified a homozygous 1-bp deletion inducing a frameshift mutation in STAG3 on chromosome 7. STAG3 encodes a meiosis-specific subunit of the cohesin ring, which ensures correct sister chromatid cohesion. Female mice devoid of Stag3 are sterile, and their fetal oocytes are arrested at early prophase I, leading to oocyte depletion at 1 week of age.

P

REMATURE OVARIAN FAILURE, THE END POINT OF PRIMARY OVARIAN insufficiency, affects approximately $1 \%$ of women worldwide. Patients with premature ovarian failure present with at least a 6-month history of amenorrhea and elevated plasma levels of follicle-stimulating hormone ( $>40 \mathrm{mIU}$ per milliliter). The disorder can result from premature depletion of the follicle pool, follicular atresia, follicle growth arrest, or ovarian dysgenesis. Although a majority of cases are idiopathic, premature ovarian failure can be caused by infectious agents, chemotherapy, pelvic surgery, autoimmune disease, environmental factors, or genetic conditions. ${ }^{1}$ The disorder is observed in syndromic diseases - for example, Turner's syndrome and BPES (blepharophimosis, ptosis, and epicanthus inversus syndrome) - or in isolated disease.

In at least 10 to $15 \%$ of patients with premature ovarian failure, a genetic cause has been determined. ${ }^{2,3}$ To date, genetic alterations including chromosomal deletions, rearrangements, and autosomal and X-linked mutations have been identified in patients with this disorder. ${ }^{2,4,5}$ Genomewide association studies have also provided insight into novel genomic regions that are important in ovarian insufficiency and failure. ${ }^{6,7}$ However, in most cases of premature ovarian failure, no genetic cause has been identified..$^{2,3,4,6}$

In analyses of samples obtained from a consanguineous Palestinian family with premature ovarian failure, we previously identified a 10-Mb region on 7q21.3-22.2 and a 3-Mb region on 7p21.1-15.3 that had significant linkage with premature ovarian failure (maximum logarithm of the odds [LOD] score, 3.26), which was consistent with homozygosity by descent. ${ }^{8}$ By combining linkage data and exome sequencing in this family, we have identified a homozygous 1-bp deletion in the gene encoding stromal antigen 3 (STAG3), a finding that is further supported by the phenotype of female mice with a homozygous disruption in Stag3.
From Institut Jacques Monod, Université Paris Diderot (S.C., M.F., R.A.V.), and Institut Cochin, Université Paris Descartes, Centre National de la Recherche Scientifique, Unité Mixte de Recherche 8104, INSERM (D.V., M.F.) — both in Paris; the Department of Human Genetics, David Geffen School of Medicine, University of California, Los Angeles, Los Angeles (V.A.A., E.V.); Departamento de Fisiología y Farmacología, Universidad de Salamanca (E.L.), and Instituto de Biología Moleculary Celular del Cáncer (E.L., I.G.-T., A.M.P.) both in Salamanca, Spain; the Department of Molecular Cellular Biology, Baylor College of Medicine, Houston (P.A.O., K.O., W.H.); Centro de Investigaciones Biológicas, Consejo Superior de Investigaciones Científicas, Madrid (J.L.B.); and the Department of Genetics, Hadassah University Hospital, Jerusalem (Z.B.-N.). Address reprint requests to Dr. Vilain at the Department of Human Genetics, University of California, Los Angeles, 695 Charles E. Young Dr., Gonda 5506, Los Angeles, CA 90095, or at evilain@ucla.edu; or to Dr. Pendás at Centro de Investigación del Cáncer, Campus Miguel de Unamuno, 37007 Salamanca, Spain, or at amp@usal.es.

Drs. Caburet, Arboleda, and Llano contributed equally to this article.

N Engl J Med 2014;370:943-9.

DOI: 10.1056/NEJMbrl309635

Copyright (C) 2014 Massachusetts Medical Society. 
CASE REPORT

The Middle Eastern Family 1 (MO1DA), whose members have been described in detail previously, ${ }^{8}$ presented at the Department of Genetics at Hadassah Hospital in Jerusalem for treatment of premature ovarian failure, the extreme end of the spectrum of primary ovarian insufficiency (Fig. 1A). The proband (Family Member IV-1) was the eldest of six sisters. The family history showed that in this consanguineous Middle Eastern family of Palestinian origin, five women presented with premature ovarian failure. Of the six sisters in generation IV, four were affected. The mother (Family Member III-1) and father (Family Member III-2) were first cousins and were linked by at least three other previous consanguineous marriages. In addition to the four sisters in generation IV, premature ovarian failure had also been diagnosed in a maternal aunt. The nonaffected sisters of the proband (Family Members IV-3 and IV-4) had menarche at ages within the normal range and were fertile; they naturally conceived two and three children, respectively. The two brothers (Family Members IV-2 and IV-5) were also fertile, with three children each.

All four affected sisters had received the diagnosis of premature ovarian failure between the ages of 17 and 20 years and initially presented with primary amenorrhea. On physical examination, they all had small and undeveloped breasts; the height was within the normal range. Ultrasonographic examination showed bilateral streak gonads (i.e., composed mainly of fibrous tissue). Hormonal studies that were performed at the time of diagnosis showed the expected high gonadotropin levels and low estradiol levels, with follicle-stimulating hormone levels of more than $45 \mathrm{mIU}$ per milliliter (normal range, 3 to $21 \mathrm{mIU}$ per milliliter), luteinizing hormone levels of more than $18 \mathrm{mIU}$ per milliliter (normal range, 1 to $18 \mathrm{mIU}$ per milliliter), and estradiol levels of less than 22 pg per milliliter (81 pmol per liter; normal range, 30 to $190 \mathrm{pg}$ per milliliter [110 to 697 pmol per liter]). We ruled out the presence of autoantibodies in all four sisters.

We performed genetic testing on samples obtained from the four sisters with the disorder to rule out known genetic causes of premature ovarian failure. The results of this testing showed a normal 46,XX karyotype and no premutations in the gene encoding fragile $\mathrm{X}$ mental retardation 1 (FMR1). At the age of 19, the youngest sister with premature ovarian failure (Family Member IV-10) was found to have simultaneous bilateral ovarian tumors consisting of a gonadoblastoma on the right ovary and a complex tumor consisting of a dysgerminoma, embryonal carcinoma, and choriocarcinoma in the left ovary. No other relevant medical finding was identified in the other family members with premature ovarian failure.

METHODS

\section{PARTICIPANTS AND GENETIC STUDIES}

We obtained written informed consent from all participants in the study. After whole-exome capture on Agilent SureSelectXT 50 Mb Exon Capture, we performed high-throughput sequencing of the DNA obtained from one participant with premature ovarian failure and from one of her unaffected sisters. We performed Sanger sequencing to confirm the segregation of the identified variants in the unaffected parents, affected sisters, and unaffected siblings (Table S2 in the Supplementary Appendix, available with the full text of this article at NEJM.org).

\section{MOUSE MODEL}

The OVE2312C mouse line, obtained from the Jackson Laboratory (Sacramento, CA), was generated with the use of a lentiposon insertional mutagenesis strategy and carries an insertion in Stag3 that leads to a null allele (Fig. S1, S3, and S5 in the Supplementary Appendix). All experiments involving animals were performed in accordance with procedures approved by the institutional animal ethics committee at the Cancer Research Center in Salamanca.

\section{RESULTS}

\section{HOMOZYGOUS MUTATION IN STAG 3}

We performed whole-exome sequencing of DNA obtained from two of the sisters, one who was affected with premature ovarian failure (Family Member IV-1) and the other who was unaffected (Family Member IV-3) (Fig. 1A). Exome sequencing generated 3.3 billion reads per sample and covered $92.6 \%$ of the targeted sequence at $10 \times$ or greater (Table S3 in the Supplementary Appendix). We filtered the single-nucleotide variants 
and insertions or deletions using the following criteria: location in the linkage peaks, homozygosity in the affected sibling, heterozygosity in the unaffected sibling, and absence from the Single-Nucleotide Polymorphism Database (dbSNP), build 132, and the Human Gene Mutation Database. Our data confirmed the large homozygous regions on chromosome 7 in Family Member IV-1 but not in Family Member IV-3. The filtering algorithm identified eight variants in six genes, all located within the 7q21.3-22.2 linkage peak. Of these six genes, five were ruled out as unlikely to harbor a causal mutation for premature ovarian failure on the basis of known function or domain of expression (Tables S4 and S5 in the Supplementary Appendix).

The remaining gene, STAG3, carried a 1-bp deletion, c.968delC. STAG3 encodes a subunit of cohesin, a large protein complex that is essential for proper pairing and segregation of chromosomes during meiosis. ${ }^{9-11}$ The deletion results in a deleterious frameshift p.F187fs ${ }^{\star} 7$ mutation and a premature stop codon, which predicts omission of exons 8 to 34 from translated STAG3 messenger RNA. The resulting transcript would either undergo nonsense-mediated decay ${ }^{12}$ or result in a severely truncated protein. In the mutant protein, the STAG domain would be compromised by truncation (Fig. 1B) and the armadillo (ARM)-type domain (which is predicted to interact with a nucleic acid or another protein) would be absent. Human STAG3 expression is restricted to early meiosis in fetal ovaries in females and from the onset of spermatogenesis in males. ${ }^{13-15}$

The STAG3 mutation was confirmed on Sanger sequencing, and the mutation cosegregated with the phenotype for premature ovarian failure (Fig. S2 in the Supplementary Appendix). The four sisters with premature ovarian failure were homozygous for the STAG3 mutation, whereas the unaffected family members who were analyzed were either heterozygous or homozygous for the nonmutant allele (Fig. 1A).

\section{INACTIVATION OF STAG 3 IN MURINE MODEL}

To confirm that the loss of function of STAG3 is associated with premature ovarian failure, we characterized the female phenotype of mice carrying an insertional null mutation in Stag3. Hemizygous mice transmitted the mutation to their offspring at mendelian frequencies (1:2:1).

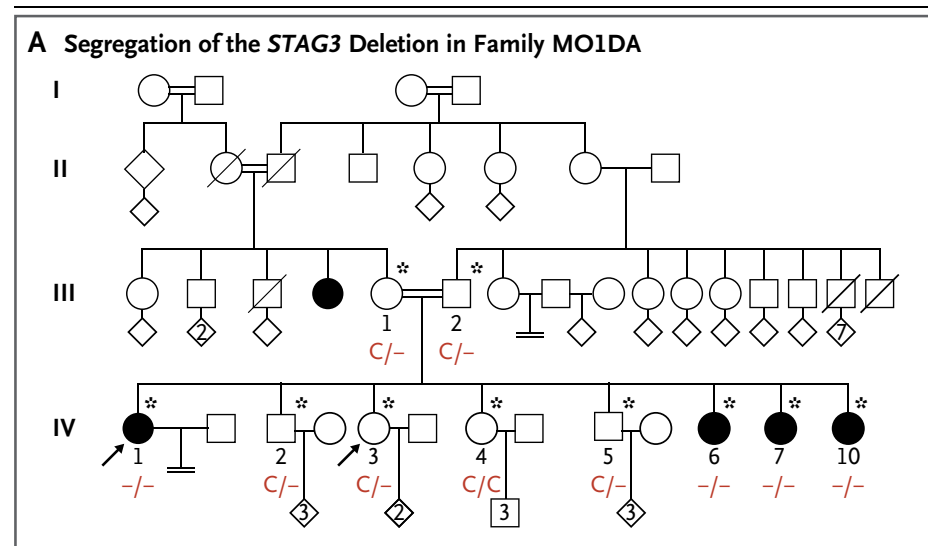

B Structure of STAG3

Exon 7:

Chr7: 99,786,435-99,786,639

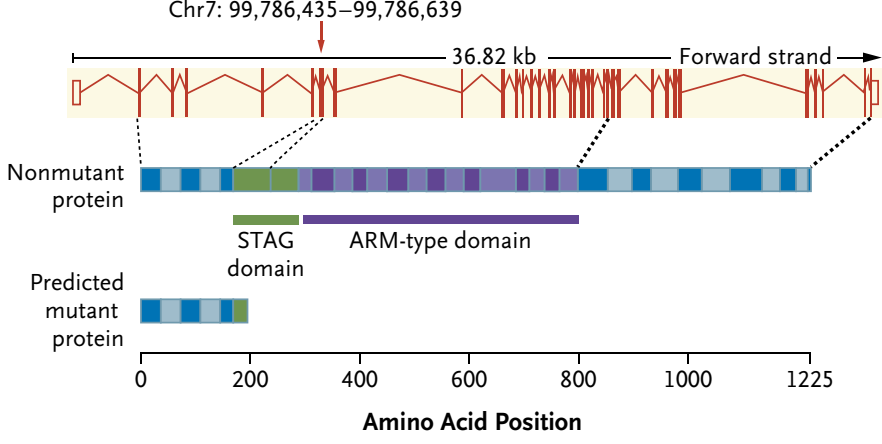

Figure 1. Identification of a Mutation in the Coding Sequence of STAG3 in a Consanguineous Family with Premature Ovarian Failure.

Panel A shows the family pedigree, with double horizontal lines indicating consanguineous unions. Squares denote male family members, circles female family members, diamonds offspring for whom information on sex was not available (with the number of children indicated inside the symbols if it was known), solid symbols affected family members, open symbols unaffected family members, and slashes deceased family members. Arrows point to the two sisters who provided samples for whole-exome sequencing. The genotypes for the mutation in STAG3 are indicated below each family member whose DNA was available for Sanger sequencing, as shown with an asterisk. Panel B shows the organization of STAG3 (from Ensembl, reference transcript ENST00000426455). Exons are depicted as red vertical bars and introns as dashed lines. The solid bars indicate coding exons, and the open bars at each end indicate noncoding exons. The structure of the nonmutant protein is shown below the exons for the longest isoform of 1225 amino acids, with coding exons shown as bars with alternating colors. The mutation lies in exon 7 (indicated by the arrow), which encodes the first part of the STAG domain (in green). The predicted length of the mutant protein (if translated) is 194 amino acids, as induced by the frameshift mutation and premature stop codon. In the mutant protein, the armadillo (ARM)-type domain, which is predicted to interact with a nucleic acid or another protein, would be absent.

Homozygous mutant mice were identified by means of the Southern blot technique (Fig. S3 in the Supplementary Appendix). The absence of both transcript and protein was shown on Northern blot analysis and immunofluorescence, indi- 
cating that the insertional mutation is a null allele (Fig. S4 and S5 in the Supplementary Appendix). STAG3 expression is restricted to the germline in mice, ${ }^{16}$ and Stag $3^{-1-}$ mice showed no overt phenotype apart from sterility.

In mouse oocytes, the depletion of genes that encode subunits of the meiotic cohesin complex induces phenotypes ranging from sterility associated with premature arrest of meiotic prophase I at a zygotene-like stage (in $\operatorname{Rec} 8)^{17}$ to subfertility caused by mild defects in the homologous chromosome synapsis at the pachytene stage (in Rad21l). ${ }^{18}$ Histologic analysis of whole ovaries of Stag $3^{-1-}$ female mice at 1,2 , and 6 weeks of age showed a distinctive lack of oocytes and ovarian follicles and a dense stroma, indicating a severe and very early ovarian dysgenesis (Fig. 2A). Since female germ cells enter meiosis during embryogenesis, ${ }^{19}$ we analyzed meiotic defects in Stag $3^{-1-}$ fetal oocytes. We analyzed oocytes from 15.5 to 19.5 gestational days (corresponding to the leptotene to diplotene stages of prophase I), using immunofluorescence staining of the SYCP3 component of the synaptonemal complex on chromosome spreads. Pachytene nuclei, characterized by 20 pairs of synapsed homologous chromosomes in control oocytes, were not observed in mutant Stag3 oocytes (Fig. 2B). The axial elements, the first parts of the synaptonemal complex to be assembled, did not progress beyond the leptotene stage in the mutant oocytes. Instead, they were very short and did not elongate to form the thin SYCP3 threads observed in late leptotene and zygotene wild-type oocytes (Fig. 2B, and Fig. S5 in the Supplementary Appendix). Stag $3^{-1-}$ oocytes did not show positive staining for SYCP1, a component of the transverse filament of the synaptonemal complex, which assembles after axial elements (Fig. 2B). We concluded that synapsis between homologues was disrupted at an early leptotene-like stage and that this premature arrest did not impair the formation of programmed doublestrand breaks or the recruitment of the RAD51 recombinase on recombination foci, because these processes appeared to be similar in normal and mutant oocytes (Fig. S6 in the Supplementary Appendix).

Because the lack of the two meiosis-specific cohesin subunits RAD21L and REC8 leads to defective loading of other cohesin subunits and defective assembly of axial elements, ${ }^{11}$ we asked whether the loss of STAG3 also compromised the loading of other cohesins in fetal oocytes. Im- munolabeling of the various meiotic cohesin subunits and SYCP3 in wild-type and Stag $3^{-1-}$ fetal ovaries showed absent or very limited colocalization of other cohesins with SYCP3 in the Stag $3^{-1-}$ mice (Fig. S7 in the Supplementary Appendix). We would therefore suggest that STAG3 forms complexes in vivo with REC8, SMC3, SMC1 $\beta$, and RAD21L, independent of the somatic or meiotic kleisin (RAD21), and that STAG3 is essential for the formation of a functional cohesin ring and synaptonemal complex in mice and humans.

In addition to its role in the formation of a synaptonemal complex, ${ }^{11,18}$ the cohesin complex holds sister chromatids together. ${ }^{10,20}$ Therefore, we analyzed centromeric sister chromatid cohesion by means of immunolocalization of centromeres in fetal oocytes. Mutant oocytes showed an obvious loss of centromeric sister chromatid cohesion (Fig. S8 in the Supplementary Appendix). These results suggest that STAG3-containing cohesin complexes also play a central role in the maintenance of sister chromatid cohesion in mammalian oocytes (Fig. S9 in the Supplementary Appendix).

DISCUSSION

Using a combination of linkage data and exome sequencing, we identified a frameshift mutation in STAG3 in a large consanguineous family with premature ovarian failure. All affected family members who provided samples for analysis were homozygous for the mutation. Our results are supported by the sterile phenotype and the very early ovarian defects observed in Stag $3^{-1-}$ female mice. The early meiotic arrest and the centromeric chromosomal cohesion defects observed in Stag $3^{-1-}$ fetal oocytes provide further evidence that Stag3 is essential for the assembly of the meiotic cohesin ring and the synaptonemal complex. Given the essential role of STAG3 in meiosis, we expect that a homozygous defect would lead to infertility in both sexes. However, since the brothers of the proband are heterozygous, we cannot draw conclusions from this family with regard to male infertility. Nevertheless, we found that male Stag $3^{-1-}$ mice were infertile (Fig. S10 in the Supplementary Appendix).

Mutations in mice that affect other meiotic cohesin subunits also result in abnormal oocyte development. Specifically, oocytes show meiotic defects because of abnormal formation of axial elements (in mutant REC8), ${ }^{17}$ impaired sister chro- 
matid cohesion leading to massive aneuploidy during the second meiotic division (in mutant SMC1 $\beta),{ }^{21}$ or mild defects in synapsis resulting in premature depletion of the oocyte pool (in mutant RAD21L). ${ }^{18}$ Furthermore, the gradual loss of meiotic cohesins or their protector, shugoshin-like 2, is an important mechanism of meiotic nondisjunction and age-related aneuploidy and infertility, as shown in mice and postulated in humans. ${ }^{22-25}$

Although some human cases of premature ovarian failure have been attributed to defective chromosomal cohesion at later stages of meiosis because of supernumerary chromosomes, ${ }^{26}$ our results show that this disorder can also result from a defect in sister chromatid cohesion much earlier, in prophase. The very few mutations that have been identified in meiotic genes in association with human infertility result in azoospermia or recurrent pregnancy loss or alter processes other than the formation of the synaptonemal complex. ${ }^{27-30}$ Genetic analyses of samples obtained from four other families with recessive inheritance of infertility ruled out linkage with the STAG3 locus, highlighting the genetic heterogeneity of this disease and suggesting that its recessive forms are infrequent (see Section I in the Supplementary Appendix).

Cohesins are implicated in the repair, replication, and recombination of DNA, as well as in chromosomal stability, transcription regulation, stem-cell pluripotency, and cell differentiation. ${ }^{10,20,31}$ Our study expands the spectrum of cohesinopathies, which include severe constitutional disorders such as the Cornelia de Lange syndrome and the Roberts syndrome, to one with isolated infertility.

In addition to having roles in crucial developmental processes, genes such as SMC1, SMC3, STAG3, STAG2, and RAD21 are mutated in a variety of cancers ${ }^{32,33}$ (see also the Catalogue of Somatic Mutations in Cancer [COSMIC] database). Indeed, somatic mutations in RAD21, SMC1A, SMC3, and STAG2 (a paralogue of STAG3) are considered to be driver mutations in acute myeloid leukemias and several solid tumors, ${ }^{34-37}$ and loss of heterozygosity in STAG3 occurs in epithelial ovarian carcinomas. ${ }^{38}$ It is worth noting that at the age of 19, the youngest sister with premature ovarian failure (Family Member IV-10) presented with bilateral ovarian cancer. The germinal origin of these tumors suggests a possible role for the germline STAG3 truncating mutation in genomic instability in the few oocytes that survived ovarian degeneration. Accordingly, it would be inter-

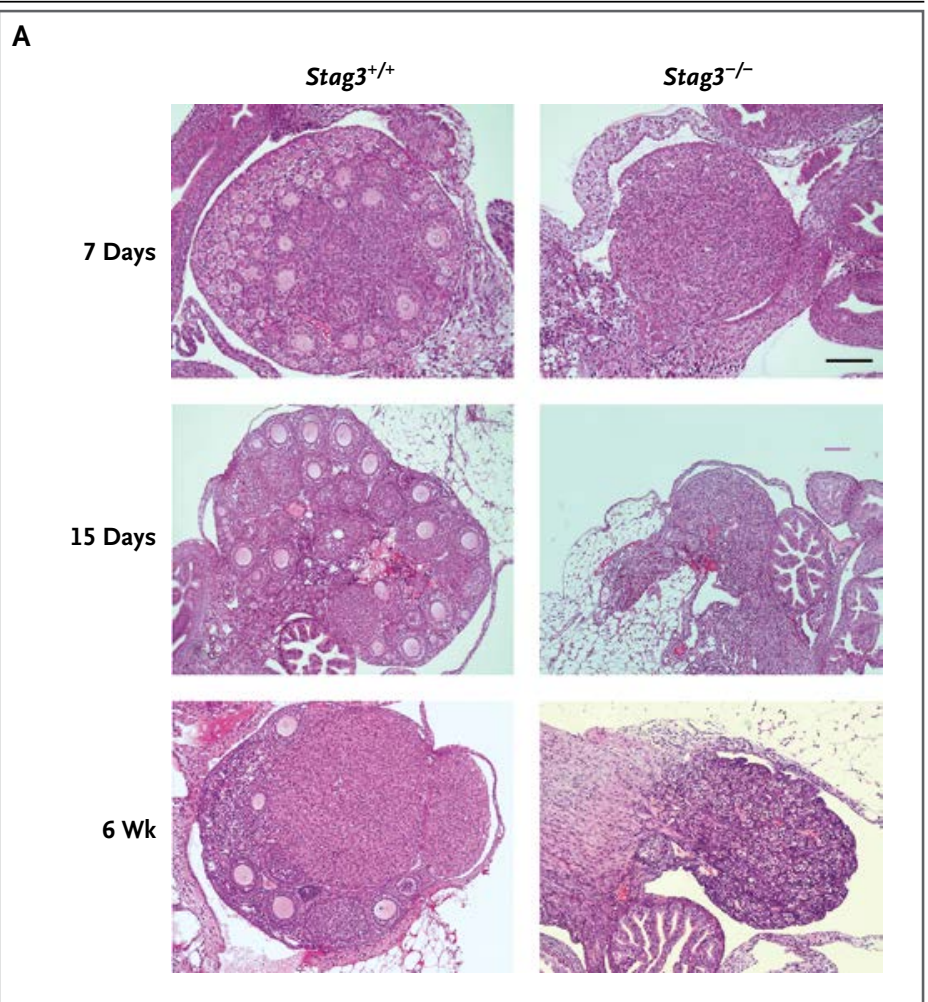

B

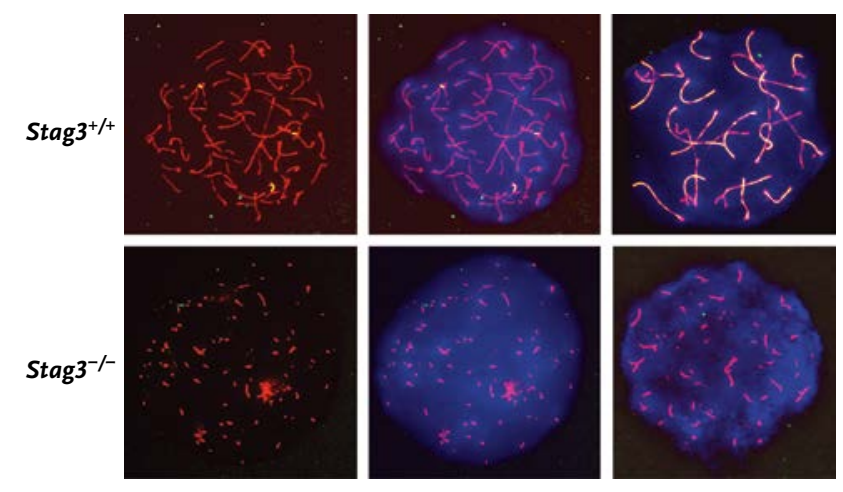

Figure 2. Premature Ovarian Failure in Stag3-1- Mice.

Panel A shows a comparative histologic analysis of ovarian sections from wild-type and Stag $3^{-1-}$ mice at 7 days, 15 days, and 6 weeks of age. Growing oocytes and follicle development are evident in wild-type animals at each age. Ovaries with no follicles or oocytes were observed in their Stag3-deficient littermates. At 6 weeks, Stag3-deficient mice showed atrophic and fibrotic ovaries. The scale bar represents $100 \mu \mathrm{m}$ in 7-day sections and $200 \mu \mathrm{m}$ in 15-day and 6-week sections. Panel B shows immunostaining assays for SYCP3 (detected in red) and SYCP1 (detected in green) in murine fetal oocytes. The yellow lines indicate the formation of synapses. SYCPI, a marker for transverse filaments of the synaptonemal complex, is located along the synapsed lateral elements in the wild-type oocyte from the early-zygotene stage (top left and middle panels) until the late-zygotene stage (top right panel), when almost all the lateral elements are synapsed. However, the Stag $3^{-1-}$ oocytes that are arrested at the leptotene-like stage (bottom panels) do not show SYCPI staining or synapsis; the top and bottom middle and right panels also show DAPI staining of DNA in blue. 
esting to investigate whether women carrying STAG3 mutations resulting in premature ovarian failure have an increased risk of germ-cell tumors. Our results implicate STAG3 mutation in premature ovarian failure and the meiosis-specific cohesin complexes in enabling fertility. Further studies are required to understand the association between genetic variants in STAG3, human infertility, and ovarian tumorigenesis. We propose that mutations affecting other meiosis-specific subunits of the cohesin complex could lead to similar functional defects and explain ovarian failure in women with premature ovarian failure.

Supported by grants from the University Paris Diderot-Paris 7 (to Drs. Caburet, Veitia, and Fellous), the Ligue Nationale contre le Cancer and the Centre National de la Recherche Scientifique (to Drs. Caburet and Veitia), the GIS (Groupement d'Intérêt
Scientifique) Institut des Maladies Rares (to Drs. Caburet and Fellous), the Doris Duke Charitable Foundation and National Institute of Child Health and Human Development (NICHD) (RO1HD068138 and R01HD044513, to Drs. Arboleda and Vilain), the NICHD (F31HD068136) and the UCLA Medical Scientist Training Program (both to Dr. Arboleda), and the Ministerio de Economía y Competitividad (SAF2011-25252) and Junta de Castilla y León (both to Drs. Llano and Pendás). The lentiviral mutagenesis studies were supported by the Ocular Development Fund (to Dr. Overbeek).

Disclosure forms provided by the authors are available with the full text of this article at NEJM.org.

We thank the proband's family members for their continuous support and participation in this research over the years, Fabien Fauchereau for his help with additional genetic analyses, the pathology unit of the Centro de Investigación del Cáncer-Salamanca, Dr. Franco DeMayo and Dong-Cai Liang for the mouse embryo manipulations, Aron Geurts for the Sleeping Beauty transposon, Gary Felsenfeld for the chicken globin insulator, Christian Rosenmund for the FUGW vector, Ningna Xiao for the confirmation of the lentiviral integration sites, and Hane Lee and Stan Nelson for their help in exome sequencing.

REFERENCES

1. Matzuk MM, Lamb DJ. The biology of infertility: research advances and clinical challenges. Nat Med 2008;14:1197-213. 2. Persani L, Rossetti R, Cacciatore C. Genes involved in human premature ovarian failure. J Mol Endocrinol 2010;45:257-79. [Erratum, J Mol Endocrinol 2010;45:405.] 3. Bachelot A, Rouxel A, Massin N, et al. Phenotyping and genetic studies of 357 consecutive patients presenting with premature ovarian failure. Eur J Endocrinol 2009;161: 179-87.

4. Shelling AN. Premature ovarian failure. Reproduction 2010;140:633-41.

5. Goswami D, Conway GS. Premature ovarian failure. Hum Reprod Update 2005; 11:391-410.

6. Zhao H, Chen ZJ. Genetic association studies in female reproduction: from candidate-gene approaches to genomewide mapping. Mol Hum Reprod 2013; 19:644-54.

7. Christin-Maitre S, Tachdjian G. Genome-wide association study and premature ovarian failure. Ann Endocrinol (Paris) 2010;71:218-21.

8. Caburet $S$, Zavadakova P, Ben-Neriah $Z$, et al. Genome-wide linkage in a highly consanguineous pedigree reveals two novel loci on chromosome 7 for nonsyndromic familial premature ovarian failure. PLoS One 2012;7(3):e33412.

9. Bardhan A. Many functions of the meiotic cohesin. Chromosome Res 2010; 18:909-24.

10. Nasmyth K. Cohesin: a catenase with separate entry and exit gates? Nat Cell Biol 2011;13:1170-7.

11. Llano E, Herrán Y, García-Tuñón I, et al. Meiotic cohesin complexes are essential for the formation of the axial element in mice. J Cell Biol 2012;197:877-85.

12. Kervestin S, Jacobson A. NMD: a multifaceted response to premature translational termination. Nat Rev Mol Cell Biol 2012; 13:700-12.

13. Houmard B, Small C, Yang L, et al. Global gene expression in the human fetal testis and ovary. Biol Reprod 2009; 81:438-43.

14. Nogués $\mathrm{C}$, Fernández $\mathrm{C}$, Rajmil $\mathrm{O}$ Templado C. Baseline expression profile of meiotic-specific genes in healthy fertile males. Fertil Steril 2009;92:578-82.

15. Garcia-Cruz R, Brieño MA, Roig I, et al. Dynamics of cohesin proteins REC8, STAG3, SMC1 beta and SMC3 are consistent with a role in sister chromatid cohesion during meiosis in human oocytes. Hum Reprod 2010;25:2316-27.

16. Pezzi N, Prieto I, Kremer L, et al STAG3, a novel gene encoding a protein involved in meiotic chromosome pairing and location of STAG3-related genes flanking the Williams-Beuren syndrome deletion. FASEB J 2000;14:581-92.

17. Xu H, Beasley MD, Warren WD, van der Horst GT, McKay MJ. Absence of mouse REC8 cohesin promotes synapsis of sister chromatids in meiosis. Dev Cell 2005;8:949-61.

18. Herrán Y, Gutiérrez-Caballero C Sánchez-Martín $M$, et al. The cohesin subunit RAD21L functions in meiotic synapsis and exhibits sexual dimorphism in fertility. EMBO J 2011;30:3091-105.

19. Peters $\mathrm{H}$. The development of the mouse ovary from birth to maturity. Acta Endocrinol (Copenh) 1969;62:98-116.

20. Mehta GD, Rizvi SM, Ghosh SK. Cohesin: a guardian of genome integrity. Biochim Biophys Acta 2012;1823:1324-42. 21. Hodges CA, Revenkova E, Jessberger R, Hassold TJ, Hunt PA. SMC1beta-deficient female mice provide evidence that cohesins are a missing link in age-related nondisjunction. Nat Genet 2005;37:1351-5.

22. Llano E, Gómez R, Gutiérrez-Caballero
C, et al. Shugoshin-2 is essential for the completion of meiosis but not for mitotic cell division in mice. Genes Dev 2008;22: 2400-13.

23. Chiang T, Duncan FE, Schindler K, Schultz RM, Lampson MA. Evidence that weakened centromere cohesion is a leading cause of age-related aneuploidy in oocytes. Curr Biol 2010;20:1522-8.

24. Lister LM, Kouznetsova A, Hyslop LA, et al. Age-related meiotic segregation errors in mammalian oocytes are preceded by depletion of cohesin and Sgo2. Curr Biol 2010;20:1511-21.

25. Nagaoka SI, Hassold TJ, Hunt PA. Human aneuploidy: mechanisms and new insights into an age-old problem. Nat Rev Genet 2012;13:493-504.

26. Bertini V, Viola D, Vitti P, Simi P, Valetto A. An idic(15) associated with POF (premature ovarian failure): molecular cytogenetic definition of a case and review of the literature. Gene 2012;503:123-5.

27. Miyamoto $T$, Hasuike $S$, Yogev L, et al. Azoospermia in patients heterozygous for a mutation in SYCP3. Lancet 2003;362:1714-9.

28. Bolor H, Mori T, Nishiyama S, et al. Mutations of the SYCP3 gene in women with recurrent pregnancy loss. Am J Hum Genet 2009;84:14-20.

29. Nishiyama $S$, Kishi T, Kato T, et al. A rare synaptonemal complex protein 3 gene variant in unexplained female infertility. Mol Hum Reprod 2011;17:266-71.

30. Mandon-Pépin B, Touraine P, Kuttenn $\mathrm{F}$, et al. Genetic investigation of four meiotic genes in women with premature ovarian failure. Eur J Endocrinol 2008; 158:107-15.

31. Remeseiro S, Losada A. Cohesin, a chromatin engagement ring. Curr Opin Cell Biol 2013;25:63-71.

32. Barber TD, McManus $\mathrm{K}$, Yuen KW, 
et al. Chromatid cohesion defects may underlie chromosome instability in human colorectal cancers. Proc Natl Acad Sci U S A 2008;105:3443-8.

33. Baudot A, de la Torre V, Valencia A. Mutated genes, pathways and processes in tumours. EMBO Rep 2010;11:805-10. 34. Ding L, Ley TJ, Larson DE, et al. Clonal evolution in relapsed acute myeloid leukaemia revealed by whole- genome sequencing. Nature 2012;481: 506-10.

35. Welch JS, Ley TJ, Link DC, et al. The origin and evolution of mutations in acute myeloid leukemia. Cell 2012;150: 264-78.

36. Solomon DA, Kim T, Diaz-Martinez LA, et al. Mutational inactivation of STAG2 causes aneuploidy in human cancer. Science 2011;333:1039-43.
37. Kon A, Shih LY, Minamino M, et al. Recurrent mutations in multiple components of the cohesin complex in myeloid neoplasms. Nat Genet 2013;45:1232-7.

38. Notaridou M, Quaye L, Dafou D, et al. Common alleles in candidate susceptibility genes associated with risk and development of epithelial ovarian cancer. Int J Cancer 2011;128:2063-74.

Copyright (c) 2014 Massachusetts Medical Society. 